Lettre de l'éditrice (Français)

Nota de la Editora (Español)

Nota de l'Editora (Català)

Editor's Note (English)

\title{
Lettre de l'éditrice (Français)
}

Suite à notre tradition de présenter en début d'année académique le travail d'un chercheur de notre Département, notre auteur invité est le docteur Xavier Fontich Vicens. Son article porte sur le défi auquel fait face à un moment ou autre tout enseignant de langues: comment enseigner la grammaire? Certains vont dire qu'il s'agit d'un des aspects plus difficiles de l'enseignement. Ceci est dû au fait de la diversité de notions qu'enseignants (et apprenants) associent au mot 'grammaire'. Pour beaucoup, le terme implique un ensemble fixe de formes et de règles d'utilisation des langues ; pour les autres, «la bonne» grammaire est associée au prestige typiquement accordé aux situations formelles d'usage oral et écrit. D'autres enseignants de langues évitent tout simplement l'enseignement de la grammaire. Trop souvent, les élèves associent grammaire avec des explications interminables de formes et de règles, suivies par des tests et des exercices. Fontich considère l'enseignement de la grammaire en classe comme un outil pour favoriser la réflexion des élèves. Encadrant son affirmation au sein d'études récentes, l'auteur propose une approche de la grammaire centrée sur le travail de groupe, dans laquelle les apprenants s'engagent dans une réflexion dialogique des textes dans leur L1.

Dans notre second article, García et Fabregat abordent l'enseignement au niveau universitaire, en particulier, dans le contexte de l'Espagne. Il devient un fait reconnu partout dans le monde que l'enseignement supérieur de qualité est directement relié à un apprentissage efficace (Delaney, Johnson, de Johnson \& Treslan, 2010). En ce sens, les auteurs présentent une étude qui examine le rôle du discours exploratoire dans les cours universitaires (en formation initiale d'enseignants) comme moyen de promouvoir l'apprentissage coopératif et ainsi assurer une plus grande qualité de l'enseignement universitaire, ainsi que la qualité des interventions des futurs enseignants dans leurs propres cours. Toujours dans l'enseignement supérieur, Zabihi, Rezazadeh et Vahid Dastjerdi portent leur regard sur la créativité des étudiants lors de l'écriture en classe d'anglais comme langue étrangère (ALE). Les auteurs comparent les résultats de l'écriture individuelle et de l'écriture collaborative, en mesurant les indices de créativité dans le processus d'écriture. Bien que les études présentées correspondent à des 
contextes différents, les auteurs coïncident dans des aspects divers avec l'article de Fontich, lorsqu'ils soulignent les avantages dérivés des tâches d'écriture coopérative.

Dans notre dernier article, Llompart adopte une approche qualitative pour observer les pratiques plurilingues de jeunes membres de familles originaires $\mathrm{du}$ Pakistan et du Maroc, vivant en Catalogne. L'auteur souligne l'importance de leurs compétences langagières hors classe et propose une vision de dimensions de l'apprentissage des langues qui ne sont pas toujours prises en considération dans les établissements scolaires. Comme Castellotti et Moore affirment, "l'expérience et les répertoires des élèves devraient être mobilisés et stimulés pour engager leur participation, et soutenir leur plurilinguisme comme un atout pour l'apprentissage et la socialisation» (Castellotti \& Moore, 2010: 5).

Le volume se termine par une révision de Mussano sur le libre d'Elaine Wilson (éditrice) intitulé School-based research. A guide for education students (Sage Publications) et un entretien avec Mercè Canals i Palau, experte en travail par projets et technologies de l'information et la communication pour l'apprentissage de langues, réalisée par Aida Guillén Onandia.

Cet ensemble de questions d'actualité apporte des idées novatrices et des perspectives intéressantes pour l'année scolaire qui commence. J'espère qu'il sera de votre intérêt.

Dr. Melinda Dooly Owenby

Août 2013

\section{Références:}

Castellotti, V., et Moore, D. (2010). Capitalising on, activating and developing plurilingual and pluricultural repertoires for better school integration. The linguistic and educational integration of children and adolescents from migrant backgrounds. Studies and Resources, No. 4. Strasbourg: Directorate of Education and Languages, DGIV. Council of Europe, Language Policy Division. Delaney, J. G., Johnson, A. N., Johnson, T. D., et Treslan, D. L. (2010). Students' perceptions of effective teaching in higher education. St. John's, NL: Distance Education and Learning Technologies.

\section{Crédits}

Les illustrations des couvertures de chaque volume ont été conçues par des étudiants de l'école EINA (Escola de Disseny i Art, Barcelona), dans le cadre du programme d'études supérieures 'Illustration créative', sous la direction de Sonia Pulido, professeure d'illustration de presse. 
Au comité de lecture du volume 6.3

L'éditrice remercie les personnes suivantes pour leur contribution à la préparation du Volume 6.3:

Necmi Akset, Cristina Aliagas, Eulalia Canals, Eva Codó, Emilee Moore, Luci Nussbaum, Adriana Patiño, Marisa Pérez, et Paul Seedhouse. 


\section{Nota de la Editora (Español)}

Los volúmenes que la nuestra revista publica al inicio de cada curso académico tienen por costumbre presentar el trabajo de un investigador del departamento que la edita. En este caso, el autor invitado es el doctor Xavier Fontich Vicens. Su artículo trata un tema que siempre presenta retos al profesorado: ¿Cómo ha de enseñarse la gramática? Seguramente estaríamos de acuerdo en que éste es uno de los aspectos de la lengua más difíciles de enseñar bien. Esto es, en parte, debido al hecho que, a menudo, el profesorado (y también el alumnado) tienen visiones muy diferentes acerca del significado del término "gramática". Hay quien cree que es un conjunto fijo de formas lingüísticas y reglas de uso. Otros defienden que la "buena" gramática es aquella que da prestigio a la comunicación oral y escrita en situaciones formales. También existe el profesorado que evitan enseñarla de manera explícita. Por otro lado, el alumnado, demasiado a menudo, asocia la enseñanza de la gramática con explicaciones interminables sobre normas de uso que han de recordarse para aprobar los exámenes. Fontich, no obstante, plantea la enseñanza de la gramática como una herramienta para promover la reflexión metalingüística en el aula. Teniendo en cuenta los estudios en este campo, el autor plantea integrar la enseñanza de la gramática en tareas de escritura realizadas en grupos. De este modo se fomenta la reflexión dialógica durante el proceso de creación de textos escritos en L1.

En nuestro segundo artículo, García and Fabrega examinan la docencia universitaria prestando especial atención al contexto español. Los autores, siguiendo las líneas de investigación en este campo, argumentan que la calidad en la educación superior está directamente relacionada con la efectividad del aprendizaje (Delaney, Johnson, Johnson, \& Treslan, 2010). Por ello, en su artículo analizan el papel que juega el habla exploratoria en aulas universitarias, concretamente en las que se llevan a cabo clases de formación inicial del profesorado, como herramienta para promover el aprendizaje cooperativo y como estrategia para mejorar la docencia en la educación superior pero también en las futuras aulas en que los maestros en formación impartirán sus clases. El artículo de Zabihi, Rezazadeh y Vahid Dastjerdi también se centra en la docencia universitaria pero analiza la creatividad de los estudiantes durante la realización de actividades de escritura en las clases de inglés como lengua extranjera. Los autores comparan los textos producidos por los alumnos a través de actividades de escritura individual y colectiva y analizan los rasgos presentes en dichos textos que 
puedan relacionarse con la escritura creativa. Aunque estos dos trabajos presentan dos contextos muy distintos al que aborda nuestro autor invitado, los autores coinciden con Fontich cuando argumentan las ventajas que presentan las tareas de escritura colaborativas para el aprendizaje de lenguas.

En nuestro último artículo, Llompart describe y analiza las prácticas comunicativas plurilingües que se desarrollan en situaciones familiares. Los informantes de dicho estudio cualitativo son miembros de familias de origen Marroquín y Paquistanís residentes en Cataluña. El reconocimiento que la autora otorga a la participación en este tipo de prácticas plurilingües como medio para el desarrollo de la competencia plurilingüe de los jóvenes de estas familias aporta datos sobre el aprendizaje de lenguas que no pueden encontrarse en contextos escolares. Tal y como afirman Castellotti y Moore "los repertorios de experiencia y conocimiento de los alumnos debería movilizarse y estimularse con el fin de animarlos a participar al tiempo que se reconoce su plurilingüismo como una baza para el aprendizaje y la socialización" (Castellotti \& Moore, 2010: 5).

Nuestro volumen se cierra con la reseña de Mussano sobre el libro editado por Elaine Wilson con el título de School-based research. A guide for education students (Sage Publications) y con la entrevista que Aida Guillén Onandia realiza a Mercè Canals Palau, quien, a partir de una experiencia personal, reflexiona sobre el uso de las TIC y la adopción del enfoque por proyectos en las aulas.

Este volumen proporciona nuevas ideas y perspectivas para tener en cuenta ahora que se inicia un nuevo curso escolar. Espero que disfrutéis con las lecturas que os proponemos.

Dr. Melinda Dooly Owenby

31 agosto de 2013

\section{Referencias Bibliográficas}

Castellotti, V., y Moore, D. (2010). Capitalising on, activating and developing plurilingual and pluricultural repertoires for better school integration. The linguistic and educational integration of children and adolescents from migrant backgrounds. Studies and Resources, No. 4. Strasbourg: Directorate of Education and Languages, DGIV. Council of Europe, Language Policy Division.

Delaney, J. G., Johnson, A. N., Johnson, T. D., y Treslan, D. L. (2010). Students' perceptions of effective teaching in higher education. St. John's, NL: Distance Education and Learning Technologies. 


\section{Créditos}

Las ilustraciones para las portadas de cada edición las diseñan los estudiantes de EINA (Escuela de Diseño y Arte, Barcelona), dentro del programa del posgrado en Ilustración Creativa, bajo la dirección de Sonia Pulido, profesora de Ilustración de Prensa.

\section{Los revisores del volumen 6.3}

La editora agradece a las siguientes personas su contribución a la preparación del Volumen 6.3:

Necmi Akset, Cristina Aliagas, Eulalia Canals, Eva Codó, Emilee Moore, Luci Nussbaum, Adriana Patiño, Marisa Pérez y Paul Seedhouse. 


\section{Nota de l'Editora (Català)}

Els volums que la nostra revista publica en començar cada curs acadèmic tenen per costum presentar el treball d'un investigador del departament que l'edita. En aquest cas, l'autor convidat és el doctor Xavier Fontich Vicens. El seu article tracta un tema que sempre presenta reptes al professorat: Com s'ha d'ensenyar la gramàtica? Segurament estaríem d'acord en què és un dels aspectes de la llengua més difícils d'ensenyar bé. Això és, en part, degut al fet que, sovint, els docents (i alumnat també) tenen visions molt diferents del què vol dir el terme "gramàtica". Hi ha qui creu que és un conjunt fix de formes lingüístiques i regles d'ús. D’altres creuen que la "bona" gramàtica és aquella que dóna prestigi a la comunicació oral i escrita en situacions formals. També hi ha professorat que eviten ensenyar-la de manera explícita. Massa sovint, l'alumnat associa l'ensenyament de la gramàtica amb explicacions interminables sobre normes d'ús que s'han de recordar per aprovar els exàmens. El Dr. Fontich planteja l'ensenyament de la gramàtica com a una eina per promoure la reflexió metalingüística a l'aula. Tenint en compte els estudis en aquest camp, l'autor planteja integrar l'ensenyament de la gramàtica en tasques d'escriptura realitzades en grups. Així es fomenta la reflexió dialògica durant el procés de creació de textos escrits en L1.

En el nostre segon article, García i Fabrega examinen la docència universitària $i$ es centren en el context espanyol. Els autors, seguint les línies d'investigació en aquest camp, argumenten que la qualitat en l'educació superior està directament relacionada amb l'efectivitat en l'aprenentatge (Delaney, Johnson, Johnson, \& Treslan, 2010). Per aquest motiu, en el seu article analitzen el paper de la parla exploratòria en les aules universitàries, concretament en les dedicades a la formació inicial del professorat, com a eina per promoure l'aprenentatge cooperatiu i com a estratègia per millorar la docència en l'educació superior però també en les futures aules en què aquests mestres en formació impartiran les seves classes. L'article de Zabihi, Rezazadeh i Vahid Dastjerdi també es centra en la docència universitària però analitza la creativitat dels estudiants durant la realització d'activitats d'escriptura a les aules d'anglès com a llengua estrangera. Els autors comparen els textos produïts pels alumnes a través d'activitats d'escriptura individual i col-lectiva i analitzen els trets que presenten aquests textos $\mathrm{i}$ que poden relacionar-se amb l'escriptura creativa. Encara que aquests dos treballs presenten dos contextos molt diferents respecte al context en què treballa el nostre autor convidat, els autors d'ambdós articles coincideixen amb Fontich quan argumenten els 
avantatges que presenten les tasques d'escriptura col-laboratives per a l'aprenentatge de llengües.

En el nostre últim article, Llompart descriu i analitza les pràctiques comunicatives plurilingües que es desenvolupen en situacions familiars. Els informants d'aquest estudi qualitatiu són membres de famílies d'origen Marroquí i Paquistanès residents a Catalunya. El reconeixement que l'autora atorga a la participació en aquest tipus de pràctiques plurilingües com a mitjà que afavoreix el desenvolupament de la competència plurilingüe dels joves d'aquestes famílies aporta dades sobre l'aprenentatge de llengües que no poden tractar-se en contextos escolares. Tal i com afirmen Castellotti i Moore "els repertoris d'experiència i coneixement dels alumnes haurien de mobilitzar-se i estimular-se per tal d'animar-los a participar, alhora que es reconeix el seu plurilingüisme com un avantatge per a l'aprenentatge i la socialització" (Castellotti \& Moore, 2010: 5).

El nostre volum conclou amb la ressenya de Mussano sobre el llibre editat per Elaine Wilson que porta per títol School-based research. A guide for education students (Sage Publications) i amb la entrevista que Aida Guillén Onandia realitza a Mercè Canals Palau, qui, a partir d'una experiència personal, reflexiona sobre l'ús de les TIC i l'adopció de l'enfocament per projectes a l'aula.

Aquest volum proporciona noves idees i perspectives per tenir en compte ara que comença un nou curs escolar. Espero que gaudiu de les lectures que us proposem.

Melinda Dooly Owenby

31 agost de 2013

\section{Referències Bibliogràfiques}

Castellotti, V., i Moore, D. (2010). Capitalising on, activating and developing plurilingual and pluricultural repertoires for better school integration. The linguistic and educational integration of children and adolescents from migrant backgrounds. Studies and Resources, No. 4. Strasbourg: Directorate of Education and Languages, DGIV. Council of Europe, Language Policy Division.

Delaney, J. G., Johnson, A. N., Johnson, T. D., i Treslan, D. L. (2010). Students' perceptions of effective teaching in higher education. St. John's, NL: Distance Education and Learning Technologies. 


\section{Crèdits}

Les il·lustracions per a les portades de cada edició són dissenyades pels estudiants d'EINA (Escola de Disseny i Art, Barcelona), dins del programa del postgrau d'Il·lustració Creativa, sota la direcció de Sonia Pulido, professora d'Il·lustració de Premsa.

\section{Els revisors de volum 6.3}

L'editora agraeix a les següents persones per la seva contribució a la preparació del Volum 6.3:

Necmi Akset, Cristina Aliagas, Eulalia Canals, Eva Codó, Emilee Moore, Luci Nussbaum, Adriana Patiño, Marisa Pérez i Paul Seedhouse. 


\section{Editor's Note (English)}

Continuing our Autumn tradition of highlighting the work of a teacher and researcher from our department, our guest author for this volume is Dr. Xavier Fontich Vicens. His article tackles a challenge that almost all language teachers have faced at one time or another: Grammar - how do I teach it? Many would argue that it is one of the more difficult aspects of language to teach well. In part, this may stem from the many different notions that teachers (and learners) associate with the word 'grammar'. For many, the term implies a fixed set of word forms and rules of usage, for others, 'good' grammar is associated with a certain prestige typically found in written and formal oral situations. Other language teachers may avoid grammar teaching altogether. Too often, students associate grammar with interminable explanations of forms and rules, followed by tests and drills. Fontich approaches the question of grammar teaching from the perspective that it can serve as a tool in the classroom to promote student metalinguistic reflection. Framing his assertion within recent literature in the field, the author proposes the integration of student-centred group work wherein the learners engage in dialogic reflection on texts in their $\mathrm{L} 1$.

In our second article, García and Fabregat look at teaching at the university level, in particular, within the context of Spain. It is becoming an acknowledged fact across the globe that quality teaching at higher education is directly connected to effective learning (Delaney, Johnson, Johnson, \& Treslan, 2010). Along these lines, the authors present a study that examines the role of exploratory talk in university classrooms (in Initial Teacher Training) as means of promoting cooperative learning and thereby ensuring more quality teaching at both university level and as part of the student-teachers' future interventions in their own classrooms. Also looking at higher education, Zabihi, Rezazadeh and Vahid Dastjerdi shift the focus to look at students' creativity when writing in English as a Foreign Language (EFL) classes. The authors compare individual and collaborative writing output by measuring specific features they relate to creativity in the writing process. Although the studies are very contextually different, these authors coincide in several aspects with Fontich's article when highlighting the advantages derived from collaborative writing tasks.

In our last article, Llompart takes a qualititative approach to look at the plurilingual practices produced in family situations of families from Pakistan and Morocco living in Catalonia. The author's tacit acknowledgement of the importance of 
the plurilingual competences of these young learners outside of the classroom ensures insight into facets of language learning that is not always explicit in school settings. As Castellotti and Moore state "pupils' experience and knowledge repertoires should be mobilized and stimulated to engage their participation, and to support their plurilingualism as an asset for learning and socialization" (Castellotti \& Moore, 2010: $5)$.

We finish this volume with Mussano's a review of Elaine Wilson's (editor) book entitled School-based research. A guide for education students (Sage Publications) and an interview of Mercè Canals i Palau, expert in project- work and ICT for language learning, carried out by Aida Guillén Onandia.

This timely issue brings innovative ideas and intriguing perspectives to contemplate as the new academic year begins. I hope you enjoy it.

Dr. Melinda Dooly Owenby

31 August 2013

\section{Works Cited:}

Castellotti, V., \& Moore, D. (2010). Capitalising on, activating and developing plurilingual and pluricultural repertoires for better school integration. The linguistic and educational integration of children and adolescents from migrant backgrounds. Studies and Resources, No. 4. Strasbourg: Directorate of Education and Languages, DGIV. Council of Europe, Language Policy Division.

Delaney, J. G., Johnson, A. N., Johnson, T. D., \& Treslan, D. L. (2010). Students' perceptions of effective teaching in higher education. St. John's, NL: Distance Education and Learning Technologies.

\section{Credits:}

Illustrations for the covers of each issue are designed by students of EINA (Escola de Disseny i Art, Barcelona) studying in the postgraduate course 'Il-lustració Creativa', under the direction of Sonia Pulido, teacher of Illustration for Publishing Media.

\section{Reviewers for Volume 6.3}

The editor would like to thank the following reviewers for their contribution to the preparation of Volume 6.3:

Necmi Akset, Cristina Aliagas, Eulalia Canals, Eva Codó, Emilee Moore, Luci Nussbaum, Adriana Patiño, Marisa Pérez, and Paul Seedhouse. 